\title{
ON LEFT DERIVATIONS AND RELATED MAPPINGS
}

\author{
M. BREŠAR AND J. VUKMAN
}

(Communicated by Palle E. T. Jorgensen)

Dedicated to Professor Ivan Vidav on his seventieth birthday

\begin{abstract}
Let $R$ be a ring and $X$ be a left $R$-module. The purpose of this paper is to investigate additive mappings $D_{1}: R \rightarrow X$ and $D_{2}: R \rightarrow X$ that satisfy $D_{1}(a b)=a D_{1}(b)+b D_{1}(a), a, b \in R$ (left derivation) and $D_{2}\left(a^{2}\right)=2 a D_{2}(a), a \in R$ (Jordan left derivation). We show, by the rather weak assumptions, that the existence of a nonzero Jordan left derivation of $R$ into $X$ implies $R$ is commutative. This result is used to prove two noncommutative extensions of the classical Singer-Wermer theorem.
\end{abstract}

\section{Preliminaries}

Throughout, $R$ will represent an associative ring with center $Z(R)$. Recall that $R$ is prime if $a R b=0$ implies $a=0$ or $b=0$, and $R$ is semiprime if $a R a=0$ implies $a=0$. A module $X$ is said to be $n$-torsionfree, where $n$ is an integer, if $n x=0, x \in X$ implies $x=0$. Let $R$ be a ring and $X$ be an $R$-bimodule. An additive mapping $D: R \rightarrow X$ is called a derivation (Jordan derivation) if $D(a b)=D(a) b+a D(b), a, b \in R \quad\left(D\left(a^{2}\right)=D(a) a+a D(a)\right.$, $a \in R)$. Obviously, every derivation is a Jordan derivation. The converse in general is not true. A well-known result of I. N. Herstein [6] states that in case $R$ is a prime ring of characteristic not 2 , then every Jordan derivation $D: R \rightarrow R$ is a derivation. A brief proof of this result in presented in our recent paper [3].

An additive mapping $D: R \rightarrow X$, where $R$ is a ring and $X$ is a left $R$ module will be called a left derivation if $D(a b)=a D(b)+b D(a), a, b \in R$. An additive mapping $D: R \rightarrow X$ will be called a Jordan left derivation if $D\left(a^{2}\right)=2 a D(a), a \in R$. It turns out that the notion of Jordan left derivations is in a close connection with so-called commuting mappings. A mapping $F$ of a ring $R$ into itself is said to be commuting on $R$ if $F(a) a=a F(a)$ for all $a \in$

Received by the editors February 16, 1989; some of the results appearing here were presented at the twenty-sixth International Symposium on Functional Equations, Sant Feliu de Guixols, Costa Brava, Spain, April 24-May 3, 1988.

1980 Mathematics Subject Classification (1985 Revision). Primary 16A12, 16A68, 16A70, 16A72, 39B70, 46H99.

Key words and phrases. Derivation, Jordan derivation, left derivation, Jordan left derivation, prime ring, semiprime ring, Banach algebra.

The authors' research was supported by the Research Council of Slovenia. 
$R$. There has been considerable interest for commuting and related mappings on prime rings. The fundamental result is due to E. Posner [10]. He proved that if a prime ring $R$ admits a nonzero derivation that is commuting on $R$, then $R$ is commutative. The analogous result was obtained for automorphisms [9]. The culminating theorems in the series of papers which are concerned with commuting and related mappings can be found in [1]. A result on some commuting mapping is presented in our forthcoming paper [4].

The main theorem of this paper states by the rather weak hypotheses that the existence of a nonzero Jordan left derivation $D: R \rightarrow X$ forces $R$ to be commutative. Several applications of this purely algebraic result are presented. Two of them can be considered as noncommutative extensions of the Singer-Wermer theorem [14]. This theorem states that every continuous (linear) derivation (or equivalently, left derivation) $D$ of a commutative Banach algebra $A$ maps $A$ into its radical. We show that this result is also true in the case where $A$ is noncommutative and $D$ is a continuous linear left derivation. Next we prove that every continuous linear Jordan derivation $D$ of a Banach algebra $A$ with the property $D(a) a-a D(a) \in \operatorname{rad}(A)$, where $\operatorname{rad}(A)$ is a radical of $A$, maps $A$ into $\operatorname{rad}(A)$. This improves a result of [15].

Finally we use our main theorem in solving some functional equations on operator algebras. The result we obtain may be of some interest from the automatic continuity point of view.

\section{LEFT JORDAN DERIVATIONS AND LEFT DERIVATIONS}

We start our investigations of Jordan left derivations with some preliminary results.

Proposition 1.1. Let $R$ be a ring and $X$ be a 2-torsionfree left $R$-module. If $D: R \rightarrow X$ is a Jordan left derivation then for all $a, b, c \in R$ :

$$
\begin{array}{ll}
1^{\circ} \cdot & D(a b+b a)=2 a D(b)+2 b D(a), \\
2^{\circ} \cdot & D(a b a)=a^{2} D(b)+3 a b D(a)-b a D(a), \\
3 . & D(a b c+c b a)=(a c+c a) D(b)+3 a b D(c)+3 c b D(a)-b a D(c)-b c D(a), \\
4^{\circ} \cdot(a b-b a) a D(a)=a(a b-b a) D(a), \\
5^{\circ} . & (a b-b a)(D(a b)-a D(b)-b D(a))=0 .
\end{array}
$$

Proof. The statement $1^{\circ}$ follows immediately from

$$
D\left(a^{2}\right)=2 a D(a)
$$

by the linearization (i.e., substituting $a+b$ for $a$ ). Let us prove $2^{\circ}$. From $1^{\circ}$ it follows

$$
\begin{aligned}
D(a(a b+b a)+(a b+b a) a) & =2 a D(a b+b a)+2(a b+b a) D(a) \\
& =2 a(2 a D(b)+2 b D(a))+2(a b+b a) D(a) \\
& =4 a^{2} D(b)+6 a b D(a)+2 b a D(a) .
\end{aligned}
$$


On the other hand we have

$$
\begin{aligned}
D(a(a b+b a)+(a b+b a) a) & =D\left(a^{2} b+b a^{2}\right)+2 D(a b a) \\
& =2 a^{2} D(b)+2 b D\left(a^{2}\right)+2 D(a b a) \\
& =2 a^{2} D(b)+4 b a D(a)+2 D(a b a) .
\end{aligned}
$$

In comparison we obtain $2 D(a b a)=2\left(a^{2} D(b)+3 a b D(a)-b a D(a)\right)$ which proves $2^{\circ}$ since $X$ is 2-torsionfree by the assumption. The linearization of $2^{\circ}$ gives $3^{\circ}$. Now we are able to prove $4^{\circ}$. Let us denote $D(a b(a b)+(a b) b a)$ by $A$. Then using $3^{\circ}$ we obtain $A=(a(a b)+(a b) a) D(b)+3 a b D(a b)+3 a b^{2} D(a)-$ $b a D(a b)-b a b D(a)$. On the other hand, since $A=D\left((a b)^{2}+a b^{2} a\right)$ and using (1) and $2^{\circ}$ we obtain $A=2 a b D(a b)+a^{2} D\left(b^{2}\right)+3 a b^{2} D(a)-b^{2} a D(a)=$ $2 a b D(a b)+2 a^{2} b D(b)+3 a b^{2} D(a)-b^{2} a D(a)$. By comparing the two expressions obtained from $A$ we have

$$
(a b-b a) D(a b)=a(a b-b a) D(b)+b(a b-b a) D(a) .
$$

Replacing $a+b$ for $b$ in (2), we have

$$
\begin{aligned}
(a b-b a) D(a b)+(a b-b a) D\left(a^{2}\right)= & a(a b-b a) D(a)+a(a b-b a) D(b) \\
& +b(a b-b a) D(a)+a(a b-b a) D(a),
\end{aligned}
$$

and according to (1) and (2) we obtain $4^{\circ}$. Let us write $a+b$ for $a$ in $4^{\circ}$. Using $4^{\circ}$ we obtain $(a b-b a) a D(b)+(a b-b a) b D(a)=a(a b-b a) D(b)+$ $b(a b-b a) D(a)$. Combining this relation with (2) we prove $5^{\circ}$. The proof of the proposition is complete.

We are now in a position to prove the main theorem of this paper. The staternent $4^{\circ}$ is the key identity in the proof of this theorem.

Theorem 1.2. Let $R$ be a ring and $X$ be a 2-torsionfree and 3-torsionfree left $R$-module. Suppose that $a R x=0$ with $a \in R, x \in X$ implies that either $a=0$ or $x=0$. If there exists a nonzero Jordan left derivation $D: R \rightarrow X$ then $R$ is commutative.

Proof. For the proof we need several steps. We start with

Lemma A. If $D(a) \neq 0$ for some $a \in R$, then $(a(a x-x a)-(a x-x a) a)^{2}=0$ for all $x \in R$.

Proof of Lemma A. Let $a \in R$ be a fixed element and let $x \mapsto x^{\prime}$ be a mapping defined by $x^{\prime}=a x-x a$. Now the statement $4^{\circ}$ of Proposition 1.1 can be written in the form

$$
x^{\prime \prime} D(a)=0 \quad \text { for all } x \in R .
$$

Since the mapping $x \mapsto x^{\prime}$ is a derivation, we have $(x y)^{\prime \prime}=x^{\prime \prime} y+2 x^{\prime} y^{\prime}+$ $x y^{\prime \prime}$ and, from (3), it follows that $\left(x^{\prime \prime} y+2 x^{\prime} y^{\prime}\right) D(a)=0$ holds for all pairs $x$, 
$y \in R$. Let us replace $y$ by $(y z)^{\prime}$ in the last relation. Then using (3) again we see that

$$
\left(x^{\prime \prime} y^{\prime} z+x^{\prime \prime} y z^{\prime}\right) D(a)=0 \quad \text { for all } x, y, z \in R .
$$

Substituting $z^{\prime}$ for $z$ in (4), we obtain $x^{\prime \prime} y^{\prime} z^{\prime} D(a)=0$. Finally, using this relation in (4) where we replace $y$ by $y^{\prime}$ we have that

$$
x^{\prime \prime} y^{\prime \prime} z D(a)=0 \quad \text { for all } x, y, z \in R \text {. }
$$

Since (5) holds for all $z \in R$ we are forced to conclude that $D(a) \neq 0$ implies $x^{\prime \prime} y^{\prime \prime}=0$ for all $x, y \in R$. In particular, $\left(x^{\prime \prime}\right)^{2}=0$ which is the assertion of the lemma.

Lemma B. If $a^{2}=0$ then $D(a)=0$.

Proof of Lemma B. Let us write $W$ for $D(a(x a y+y a x) a)$. The statement $2^{\circ}$ in Proposition 1.1 yields.

$$
W=a^{2} D(x a y+y a x)+3 a(x a y+y a x) D(a)-(x a y+y a x) a D(a) .
$$

Since $a^{2}=0$, we also have $0=D\left(a^{2}\right)=2 a D(a)$. We have assumed that $X$ is 2-torsionfree and so $a D(a)=0$. Hence (6) reduces to

$$
W=3 \operatorname{axay} D(a)+3 a y a x D(a) .
$$

From $2^{\circ}$ in Proposition 1.1 we have $D($ aya $)=3$ ay $D(a)$. According to $3^{\circ}$ in Proposition 1.1 we see that

$$
\begin{aligned}
W= & D(\text { ax }(a y a)+(\text { aya } x a) \\
= & \left(a^{2} y a+a y a^{2}\right) D(x)+3 a x(3 a y D(a)) \\
& +3 \text { ayax } D(a)-x a(3 a y D(a))-x a y a D(a)
\end{aligned}
$$

and since $a^{2}=0$ we have

$$
W=9 \operatorname{axay} D(a)+3 \operatorname{ayax} D(a) .
$$

By comparing (7) and (8) we arrive at $6 \operatorname{axay} D(a)=0$ and, since $X$ is 2torsionfree and 3-torsionfree, we finally have $\operatorname{axay} D(a)=0$, where $x$ and $y$ are arbitrary elements from $R$. Therefore, either $a=0$ or $D(a)=0$. In any case $D(a)=0$.

Lemma C. $R$ is commutative.

Proof of Lemma C. Take $a \in R$ such that $D(a) \neq 0$. From Lemmas A and B it follows immediately in this case that

$$
D(a(a x-x a)-(a x-x a) a)=0 \quad \text { for all } x \in R .
$$

Using $1^{\circ}$ and $2^{\circ}$ in Proposition 1.1 we obtain from (9) and the identity $a(a x-x a)-(a x-x a) a=a^{2} x+x a^{2}-2 a x a$ that

$$
\begin{aligned}
0 & =D\left(a^{2} x+x a^{2}\right)-2 D(a x a) \\
& =2 a^{2} D(x)+4 x a D(a)-2\left(3 a x D(a)+a^{2} D(x)-x a D(a)\right) \\
& =6(x a-a x) D(a) .
\end{aligned}
$$


Thus $(x a-a x) D(a)=0$ for all $x \in R$. But then for all $x, y \in R$ we have $0=((y x) a-a(y x)) D(a)=y(x a-a x) D(a)+(y a-a y) x D(a)=(y a-a y) x D(a)$. Now, since we have assumed that $D(a) \neq 0$ it follows immediately that $a \in$ $Z(R)$. Thus we have proved that $R$ is the union of its subsets $Z(R)$ and $\operatorname{Ker} D=\{a \in R \mid D(a)=0\}$. Obviously, both subsets are additive subgroups of $R$, however, a group cannot be the union of two proper subgroups. Hence $R=Z(R)$ or $R=\operatorname{Ker} D$. By the assumption $D \neq 0$ and so $R=Z(R)$; that is, $R$ is commutative. The proof of the theorem is complete.

We feel that in Theorem 1.2 the assumption that $X$ is 3-torsionfree can be avoided but we are unable to prove the result without this requirement.

The corollary below is an immediate consequence of Theorem 1.2.

Corollary 1.3. Let $R$ be a prime ring of characteristic different from 2 and 3 . If $R$ admits a nonzero Jordan left derivation $D: R \rightarrow R$ then $R$ is commutative.

As a special case of Corollary 1.3 we obtain the following result.

Corollary 1.4. Let $R$ be a noncommutative prime ring of characteristic different from 2 and 3. Let $f: R \rightarrow R$ and $D: R \rightarrow R$ be mappings. Suppose that $D$ is additive, $D\left(x^{2}\right)=D(x) f(x)+x D(x), x \in R$ and $D(x) f(x)=x D(x)$, $x \in R$. Then $D=0$. In particular, if $g: R \rightarrow R$ is a Jordan homomorphism (i.e., $g$ is additive and $g\left(x^{2}\right)=g(x)^{2}, x \in R$ ) and $a \in R$ is such that $(a g(x)-x a) g(x)=x(a g(x)-x a), x \in R$ then $a g(x)=x a, x \in R$.

Proof. The first statement is immediate. If $g$ is a Jordan homomorphism then the mapping $D: R \rightarrow R, D(x)=a g(x)-x a$ satisfies $D\left(x^{2}\right)=D(x) g(x)+$ $x D(x)$.

As we mentioned above E. Posner [10] proved that zero is the only derivation on a noncommutative prime ring $R$, which is commuting on $R$. Combining this result with the fact that every Jordan derivation on a prime ring of characteristic not 2 is a derivation, one obtains that zero is the only Jordan derivation on a noncommutative prime ring $R$ of characteristic not 2, which is commuting on $R$. This result is related to Corollary 1.4 (take a mapping $f$ in Corollary 1.4 to be the identity on $R$ ), but unfortunately we cannot say that this is a special case of Corollary 1.4 since we have the additional assumption that the ring is of characteristic not 3 .

So far we have purely algebraic results. Our next result is from analysis.

Corollary 1.5. Let $X$ and $L(X)$ be a Hausdorff locally convex vector space and the algebra of all continuous linear operators of $X$ into itself, respectively. Let $D$ be a Jordan left derivation which maps $L(X)$ either in $X$ or in $L(X)$. In both cases $D=0$.

Proof. Let us first assume that $D$ maps $L(X)$ into $X$. We shall consider $X$ as a left $L(X)$-module (multiplication by $A \in L(X)$ is operator action on $X$ ). We intend to prove that $A L(X) e=0$ implies that either $A=0$ or $e=0$. 
Let us assume that $A \neq 0$ and $e \neq 0$ in spite of the fact that $A L(X) e=0$. We have $A u \neq 0$ for some $u \in X$. There exists a continuous linear functional $f$ such that $f(e) \neq 0$ (recall that $X$ is assumed to be a Hausdorff space). Then $A(u \otimes f) e \neq 0 \quad(u \otimes f$ is defined by $(u \otimes f) x=f(x) u)$, contrary to the assumption. Hence, since all the requirements of Theorem 1.2 are fulfilled we can conclude that the proof for the case $D$ maps $L(X)$ into $X$ is complete. Using similar arguments as before one can prove that $L(X)$ is a prime ring. Hence, in case $D$ maps $L(X)$ into itself we have a special case of Corollary 1.3 .

The following simple example shows that Corollary 1.3 cannot be extended to semiprime rings. Take $R_{1}$ to be a noncommutative prime ring and $R_{2}$ to be a commutative prime ring that admits a nonzero derivation $d: R_{2} \rightarrow R_{2}$. Then $R=R_{1} \oplus R_{2}$ is a noncommutative semiprime ring and a mapping $D: R \rightarrow R$, $D\left(r_{1}, r_{2}\right)=\left(0, d\left(r_{2}\right)\right)$ is a nonzero Jordan left derivation. Note also that $D$ is in fact a derivation that maps $R$ into its center. This example leads us to the following question: Does every Jordan left derivation map $R$ into $Z(R)$ ? In the proposition below we prove that the answer to this question is affirmative in the case $D$ is a left derivation. We also show that the requirement, $X$ is 2-torsionfree and 3-torsionfree in Theorem 1.2, can be removed if $D$ is a left derivation.

Proposition 1.6. Let $R$ be a ring, $X$ be a left $R$-module, and $D: R \rightarrow X$ be a left derivation.

(i) Suppose that $a R x=0$ with $a \in R, x \in X$ implies $a=0$ or $x=0$. If $D \neq 0$ then $R$ is commutative.

(ii) Suppose that $X=R$ is a semiprime ring. Then $D$ is a derivation which maps $R$ into its center.

Proof. Consider $D(a b a), a, b \in R$. On the one hand we have

$$
D(a(b a))=a D(b a)+b a D(a)=a b D(a)+a^{2} D(b)+b a D(a) .
$$

On the other hand,

$$
D((a b) a)=a b D(a)+a D(a b)=a b D(a)+a^{2} D(b)+a b D(a) .
$$

Comparing the two expressions for $D(a b a)$ we arrive at $(a b-b a) D(a)=0$ for all $a, b \in R$. Now using $c b$ for $b$ in the last relation, we have $0=$ $(a(c b)-(c b) a) D(a)=(a c-c a) b D(a)+c(a b-b a) D(a)=(a c-c a) b D(a)$. Thus

$$
(a c-c a) b D(a)=0 \quad \text { for all } a, b, c \in R .
$$

(i) From (10) it follows immediately that for each $a \in R$ either $a \in Z(R)$ or $D(a)=0$. But then, since $Z(R)$ and $\operatorname{Ker} D=\{a \in R \mid D(a)=0\}$ are additive subgroups of $R$ we have either $R=Z(R)$ or $R=\operatorname{Ker} D$. Thus, if $D \neq 0$ then $R$ is commutative. 
(ii) Now let $X=R$ be a semiprime ring. A linearization of (10) gives $(a c-c a) b D(d)+(d c-c d) b D(a)=0$. Hence for all $a, b, c, d, x \in R$ we have

$$
\begin{aligned}
((a c-c a) b D(d)) x((a c-c a) b D(d)) & =-(a c-c a) b D(d) x(d c-c d) b D(a) \\
& =0
\end{aligned}
$$

by (10). Since $R$ is semiprime this relation yields $(a c-c a) b D(d)=0$. In particular, $(a D(d)-D(d) a) b(a D(d)-D(d) a)=0$ which implies $a D(d)=$ $D(d) a$ by the semiprimeness of $R$. That is, $D(d) \in Z(R)$ for every $d \in R$. Consequently $D$ is a derivation. The proof of the proposition is complete.

Corollary 1.7. Let $R$ be a ring, $X$ be a left $R$-module and $D: R \rightarrow X$ be a Jordan left derivation. Suppose that ax $=0$ with $a \in R, x \in X$ implies $a=0$ or $x=0$. If $D \neq 0$ then $R$ is commutative.

Proof. From $5^{\circ}$ in Proposition 1.1 we have that for each pair $a, b \in R$ either $a b=b a$ or $D(a b)=a D(b)+b D(a)$. Given $a \in R$, let $G_{a}=\{b \in R \mid a b=b a\}$ and $H_{a}=\{b \in R \mid D(a b)=a D(b)+b D(a)\}$. We see that $R$ is the union of its additive subgroups $G_{a}$ and $H_{a}$. Hence $R=G_{a}$ or $R=H_{a}$. In other words, $R$ is the union of its subsets $G=\left\{a \in R \mid G_{a}=R\right\}=Z(R)$ and $H=\left\{a \in R \mid H_{a}=R\right\}=\{a \in R \mid D(a b)=a D(b)+b D(a)$ for all $b \in R\}$. Clearly $G$ and $H$ are additive subgroups of $R$, hence $G=R$ or $H=R$. If $G=R$ then $R$ is commutative. If $H=R$ then $D$ is a left derivation and $R$ is commutative by (i) in Proposition 1.6. Thus, in any case $R$ is commutative.

\section{NonCOMmUtATIVE EXTENSIONS OF SiNGER-WERMER THEOREM}

In this section by $\operatorname{rad}(A)$ we denote the radical of an algebra $A$.

Theorem 2.1. Let $A$ be a Banach algebra. Every continuous linear left derivation $D: A \rightarrow A$ maps $A$ into its radical.

Proof. In [11] A. M. Sinclair proved that every continuous derivation of a Banach algebra $A$ leaves the primitive ideals of $A$ invariant. Using arguments similar to Sinclair's, we now show that the same is true for left derivations. Let $P$ be any two-sided ideal of $A$ and $q_{P}$ be the natural quotient map from $A$ to $A / P$. A simple modification of the proof of Lemma 2.1 in [11] shows that

$$
q_{P}\left(D^{n}\left(x^{n}\right)\right)=q_{P}\left(n ! D(x)^{n}\right)
$$

holds for every $x \in P$ and every integer $n$. Hence, if $P$ is closed then we have

$$
\begin{aligned}
\left\|\left(q_{P}(D(x))\right)^{n}\right\|^{1 / n} & =(n !)^{-1 / n}\left\|q_{P}\left(D^{n}\left(x^{n}\right)\right)\right\|^{1 / n} \\
& \leqq(n !)^{-1 / n}\|D\|\|x\| \rightarrow 0 \quad \text { as } n \rightarrow \infty .
\end{aligned}
$$

It is easy to see that $D(P)+P$ is a left ideal of $A$, hence $q_{P}(D(P))$ is a left ideal of $A / P$. From (11) it follows that $q_{P}(D(P))$ is a topologically nil left ideal, therefore, it is contained in the radical of $A / P$. Hence, if $P$ is primitive then $A / P$ is semisimple and so $q_{P}(D(P))=0$. That is, $D$ leaves $P$ invariant. 
Now, if $P$ is any primitive ideal, we may define a mapping $D_{P}$ of $A / P$ into itself by $D_{P}\left(q_{P}(x)\right)=q_{P}(D(x))$. Of course, $D_{P}$ is a linear left derivation. By the result of B. E. Johnson [7] zero is the only linear derivation of a commutative semisimple Banach algebra (see also [2, p. 95]). Thus, if $A / P$ is commutative then $D_{P}=0$ since $A / P$ is semisimple. On the other hand, if $A / P$ is noncommutative then $D_{P}=0$ by Theorem 1.2 (or Proposition 1.6) since $A / P$ is prime. In any case $D_{P}=0$ which means that $D(A)$ is contained in every primitive ideal $P$ of $A$, hence $D(A)$ is contained in $\operatorname{rad}(A)$. The proof of the theorem is complete.

It would be interesting to know whether Theorem 2.1 also holds in the case $D$ is a Jordan left derivation.

B. Yood [15] proved that every continuous linear derivation $D$ of a Banach algebra $A$ which satisfies $D(x) y-y D(x) \in \operatorname{rad}(A)$ for all $x, y \in A, \operatorname{maps} A$ into $\operatorname{rad}(A)$. We now generalize Yood's result as follows.

Theorem 2.2. Let $A$ be a Banach algebra and $D: A \rightarrow A$ be a continuous linear Jordan derivation. If $D(x) x-x D(x) \in \operatorname{rad}(A)$ for all $x \in A$, then $D$ maps $A$ into its radical.

Proof. By Lemma 3.2 in Sinclair's paper [12] every continuous linear Jordan derivation $D$ of a Banach algebra $A$ leaves the primitive ideals of $A$ invariant. Hence there is no loss of generality in assuming that $A$ is semisimple. Let $P$ be any primitive ideal of $A$. As in the proof of Theorem $2.1, D$ may be dropped to a Jordan derivation $D_{P}$ of $A / P$. Since $D_{P}(x) x=x D_{P}(x)$ holds for every $x \in A / P$ we see that $D_{P}$ is a Jordan left derivation. If $A / P$ is noncommutative then $D_{P}=0$ by Theorem 1.2. Suppose that $A / P$ is commutative. From $1^{\circ}$ in Proposition 1.1 it follows easily that $D_{P}$ is a derivation and so by the result of B. E. Johnson [7] $D_{P}=0$. Thus $D_{P}=0$ in any case, hence $D$ maps $A$ into its radical. The proof of the theorem is complete.

\section{SOME FUNCTIONAL EQUATION ON OPERATOR ALGEBRAS}

The following result was motivated by the work of S. Kurepa [8].

Theorem 3.1. Let $X$ be a Banach space and $L(X)$ be the algebra of all bounded linear operators of $X$ into itself. Let $f$ and $g$ be additive mappings which map $L(X)$ either in $X$ or in $L(X)$. Suppose that

$$
f(A)=A^{2} g\left(A^{-1}\right)
$$

is fulfilled for all invertible operators $A \in L(X)$. In this case the following assertions hold:

(i) $f(A)=g(A)$ for all $A \in L(X)$.

(ii) $f(A)=A f(\mathrm{I})$ for all $A \in L(X)$, where I denotes the identity operator.

Let us point out that in Theorem 3.1 we have two mappings in one equation and we obtain the full description of both mappings. Besides, we obtain as a 
result the continuity of both mappings under purely algebraic assumptions concerning these mappings. Therefore Theorem 3.1 might be of some interest from the automatic continuity point of view. For the results concerning automatic continuity we refer to $[5,13]$ where further references can be found.

Proof of Theorem 3.1. From (13) it follows immediately that

$$
g(A)=A^{2} f\left(A^{-1}\right)
$$

holds as well. Let us introduce a mapping $D$ by $D=f-g$. From (13) and (14) one obtains that

$$
D(A)=-A^{2} D\left(A^{-1}\right)
$$

is fulfilled for all invertible operators $A \in L(X)$. We intend to prove that $D$ is a Jordan left derivation. Since $D$ is additive it remains to prove that additivity and (15) imply that

$$
D\left(A^{2}\right)=2 A D(A)
$$

holds for all $A \in L(X)$. Let $A \in L(X)$ be arbitrary. One can choose such an integer $n$ that $B^{-1}$ and $(\mathrm{I}-B)^{-1}$ exist, where $B=n \mathrm{I}+A$. In this case we have

$$
B^{2}=B-\left(B^{-1}+(\mathrm{I}-B)^{-1}\right)^{-1} .
$$

Then from the additivity of $D$ and from (15) it follows that

$$
\begin{aligned}
D\left(B^{2}\right) & =D(B)-D\left(\left(B^{-1}+(\mathrm{I}-B)^{-1}\right)^{-1}\right) \\
& =D(B)+\left(B^{-1}+(\mathrm{I}-B)^{-1}\right)^{-2} D\left(B^{-1}+(\mathrm{I}-B)^{-1}\right) \\
& =D(B)-(\mathrm{I}-B)^{2} B^{2} B^{-2} D(B)-B^{2}(\mathrm{I}-B)^{2}(\mathrm{I}-B)^{-2} D(\mathrm{I}-B) \\
& =D(B)-(\mathrm{I}-B)^{2} D(B)+B^{2} D(B)=2 B D(B) .
\end{aligned}
$$

Hence $D\left(B^{2}\right)=2 B D(B)$ which implies $(16)$ since $D(I)=0$. Now we know that $D$ is a Jordan left derivation. From Corollary 1.5 it follows that $D=0$. Hence $f(A)=g(A)$ for all $A \in L(X)$ which proves the first part of the theorem. The relation (13) can now be written in the form

$$
f(A)=A^{2} f\left(A^{-1}\right) .
$$

It remains to prove (ii). Let us first assume that $f(\mathrm{I})=0$. Our goal is to show that in this case $f=0$. Take arbitrary operator $A \in L(X)$, and let us again choose such an integer $n$ that $B^{-1}$ and $(\mathrm{I}-B)^{-1}$ exist, where $B=n \mathrm{I}+A$. Hence $f(A)=0$ will be proved by proving that $f(B)=0$. Now according to (17) we have

$$
\begin{aligned}
f(B) & =B^{2} f\left(B^{-1}\right)=B^{2} f\left(B^{-1}(\mathrm{I}-B)\right) \\
& =B^{2}\left(B^{-1}(\mathrm{I}-B)\right)^{2} f\left((\mathbf{I}-B)^{-1} B\right) \\
& =(\mathbf{I}-B)^{2} f\left((\mathbf{I}-B)^{-1}-\mathbf{I}\right) \\
& =(\mathrm{I}-B)^{2}\left((\mathrm{I}-B)^{-1}\right)^{2} f(\mathrm{I}-B)=-f(B) .
\end{aligned}
$$


Hence $f(B)=0$ which implies $f(A)=0$, where $A \in L(X)$ is arbitrary. Let us introduce a mapping $h$ by $h(A)=f(A)-A f(\mathrm{I})$. It is obvious that $h$ is additive. A routine calculation shows that $h(A)=A^{2} h\left(A^{-1}\right)$ holds for all invertible operators $A \in L(X)$. Since $h(\mathrm{I})=0$ we have $h(A)=0$ for all $A \in L(X)$. In other words, $f(A)=A f(\mathrm{I})$ for all $A \in L(X)$ which proves (ii). The proof of the theorem is complete.

\section{REFERENCES}

1. H. E. Bell and W. S. Martindale, Centralizing mappings of semiprime rings, Canad. Math. Bull. 30 (1987), 92-101.

2. F. F. Bonsall and J. Duncan, Complete normed algebras, Springer-Verlag, 1973.

3. M. Brešar and J. Vukman, Jordan derivations on prime rings, Bull. Austral. Math. Soc. 37 (1988), 321-322.

4. - On some additive mappings in rings with involution, Aequationes Math. 38 (1989), $178-185$.

5. H. G. Dales, Automatic continuity, Bull London Math. Soc. 10 (1978), 129-183.

6. I. N. Herstein, Jordan derivations on prime rings, Proc. Amer. Math. Soc. 8 (1957), 11041110.

7. B. E. Johnson, Continuity of derivations on commutative Banach algebras, Amer. J. Math. 91 (1969), 1-10.

8. S. Kurepa, The Cauchy functional equation and scalar product in vector spaces, Glas. Mat.Fiz. Astr. 19 (1964), 23-36.

9. J. Mayne, Centralizing automorphisms of prime rings, Canad. Math. Bull. 19 (1976), 113115.

10. E. Posner, Derivations in prime rings, Proc. Amer. Math. Soc. 8 (1957), 1093-1100.

11. A. M. Sinclair, Continuous derivations on Banach algebras, Proc. Amer. Math. Soc. 20 (1969), 166-170.

12. J_ Jordan homomorphisms and derivations on semisimple Banach algebras, Proc. Amer. Math. Soc. 24 (1970), 209-214.

13. _ Automatic continuity of linear operators, London Math. Soc. Lecture Note Ser. 21, Cambridge University Press, Cambridge, London, New York, and Melbourne, 1976.

14. I. M. Singer and J. Wermer, Derivations on commutative normed algebras, Math. Ann. 129 (1955), 260-264.

15. B. Yood, Continuous homomorphisms and derivations on Banach algebras, Contemp. Math., 32, Amer. Math. Soc., Providence, RI, 1984, pp. 279-284.

Institute of Mathematics, Physics and Mechanics, P. O. Box 543, 61001 Ljubluana, YugosLavia

University of Maribor, VeKŠ, Razlagova 14, 62000 Maribor, YugoslaVia 